\title{
Biogenéricos: Un Estudio de Vigilancia Tecnológica para el Caso de la Situación en Chile*
}

\author{
Claudio Narváez Hurtado ${ }^{1}$
}

\begin{abstract}
The activities to know the market of a technological product must know not only the exclusively economic analyses but to consider the technological existing information. The present work was approached as a study of case for the application of some tools of technological alertness to determine the possibility of producing medicines of biotechnological base in Chile. Of the information that exist, is clear that the biogenerics in general are very sophisticated and need for the elaboration, a long period of management, of technical and clinical precise and sophisticated controls and significant amounts of investment in research and development. In Chile, $100 \%$ of the imported biotechnological products are finished products and that does not exist exportation of biotechnological products from Chile. In Chile yet does not have sufficient technological aptitude to include all the stages that are needed to place a biotechnological product on the market.
\end{abstract}

Keywords: Biopharmaceuticals; biogenerics; technology alertness; recombinant proteins; technological capabilities.

\section{Resumen}

Las actividades para conocer el mercado de un producto tecnológico deben conocer no solo los análisis exclusivamente económicos sino que considerar la información tecnológica existente. El presente trabajo se abordó como un estudio de caso para la aplicación de algunas herramientas de vigilancia tecnológica para determinar la posibilidad de producir medicamentos de base biotecnológica en Chile. De la información recogida se desprende que los biogenéricos en general son muy sofisticados y requieren para su elaboración, un largo período de gestión, de controles técnicos y clínicos precisos y sofisticados y montos significativos de inversión en investigación y desarrollo. En Chile, el $100 \%$ de los biofármacos importados son productos terminados y que no existe exportación de biofármacos desde Chile. Se concluye que Chile aún no tiene suficiente capacidad tecnológica para abarcar todas las etapas que se requieren para colocar un producto biotecnológico en el mercado.

Palabras claves: Biofármacos; biogenéricos; vigilancia tecnológica; proteínas recombinantes; capacidades tecnológicas.

\footnotetext{
* El siguiente trabajo es parte de una tesis del autor para optar al grado de magister en gestión tecnológica de la Universidad de Talca, Chile.

${ }^{1}$ Examinador de Patentes Industriales, Área Biotecnología. Instituto Nacional de Propiedad Industrial (www.inapi.cl). Moneda 970, piso II, Santiago. Teléfono +56-2-83603 I5. E-mail: cnarvaez@inapi.cl
}

ISSN: 07I8-2724. (http://www.jotmi.org)

Journal of Technology Management \& Innovation (c) Universidad Alberto Hurtado, Facultad de Economía y Negocios 


\section{EI Caso de los Medicamentos Biotecnológicos}

A finales de los años 70 comenzaron a patentarse los genes aislados, los procesos de obtención y las aplicaciones de productos biotecnológicos a base de ingeniería genética, con los que se obtuvieron las primeras proteínas recombinantes. La Insulina fue la primera representante de estos productos y después vinieron, la Hormona del Crecimiento, el Interferón y la Eritropoyetina. Todos estos productos son el resultado de la aplicación de la tecnología del ADN recombinante para su producción, complementada con un conjunto de áreas de investigación y desarrollo que abarcan desde la Biología molecular a los estudios clínicos.

En estos últimos años se ha planteado un nuevo foco en el mercado farmacéutico mundial relacionado con estos primeros Biofármacos (productos farmacéuticos de base biotecnológica) a los cuales se les han expirado las patentes industriales que los protegían. Estos nuevos productos denominados "Biogenéricos" o "productos biotecnológicos liberados de patentes" (Off-Patent Biotechnology Products) ya están comenzando a entrar al mercado farmacéutico (Dominguez-Gil, 2004; Schellekens, H. y Ryff, J.C., 2002).

En Chile, por otra parte, se abre una nueva oportunidad de crecimiento del mercado farmacéutico producto de la aplicación desde el año 2005, del Plan AUGE (Aseguramiento Universal con Garantías Explícitas en Salud) que garantiza por ley la atención de salud oportuna, de calidad y con protección financiera de un conjunto de enfermedades, incluidas las más graves y de mayor costo, en sus diversas etapas. Esto ya impulsó el crecimiento de las ventas de la Industria farmacéutica en alrededor de un $9 \%$ para el año 2005, a diferencia del promedio del $5 \%$ que había tenido en los años recientes. El Estado de Chile, en tanto deberá incrementar el gasto público en medicamentos, desde USD 130 millones a USD 180 millones por año (Harris, 2007). En particular, los productos de origen recombinante, tales como el interferón alfa, interferón beta, interleucina-2, son biofármacos que tienen bonificación fiscal en el caso de enfermedades catastróficas. Es decir que, considerando que la salud y la medicina constituyen elementos determinantes en la calidad de vida de las personas, el acceso a los medicamentos es de alto impacto social, y los gobiernos

ISSN: 07/8-2724. (http://www.jotmi.org)

Journal of Technology Management \& Innovation ( U Universidad Alberto Hurtado, Facultad de Economía y Negocios deben tomar medidas que permitan reducir los precios de los mismos.

Se tiene claro que los laboratorios farmacéuticos nacionales no destinan fondos a investigación y desarrollo, debido principalmente a los altos montos de inversión y tecnología que se requieren para ello. En contrapartida, estos se dedican principalmente a la fabricación de productos genéricos, con un uso de cerca del $95 \%$ de las materias primas de origen importado.

Además, dado lo pequeño del mercado local chileno, las exportaciones han pasado a ser cada vez más importantes para los fabricantes locales de medicamentos y en el año 2004, generaron ingresos por aproximadamente USD 70 millones. Laboratorios Chile, por ejemplo, ha invertido en Perú y en Argentina y exporta a más de 15 países latinoamericanos, mientras que Laboratorios Andrómaco, también opera en diversos otros países a través de la región (Harris, 2007).

En un estudio realizado por el Premio Nobel de Medicina de 1952, J. Lederberg (Lederberg, 2002) en el que se reunieron las opiniones de connotados científicos de Canadá, se elaboró un listado de las 10 biotecnologías mas promisorias, que en forma realista podrían implementarse en un lapso de 5 a 10 años en los países en desarrollo. En el estudio se indica que las enfermedades no transmisibles, tales como afecciones cardíacas y diabetes, suman más del $60 \%$ de todas las muertes en los países en desarrollo, y se espera que alcancen el $73 \%$ de todas las muertes en el 2020. Para estas enfermedades se indicó que las tecnologías del ADN recombinante para producir productos terapéuticos (insulina, interferón, etc.), es decir, los biofármacos era la biotecnología adecuada para enfrentar esta problemática.

Como país, Chile esta buscando posibilidades de mejorar la competitividad de su economía a través de su cuerpo productivo, su industria, para lo cuál se están buscando áreas de interés que permitan generar productos de alto valor agregado y que potencien el crecimiento de las capacidades tecnológicas que se han estado desarrollando. La producción de biofármacos, en especial los biogenéricos es una oportunidad que debe ser evaluada, ya sea en función de la capacidad tecnológica existente, del interés público generado por la demanda que el estado requiere de producir medicamentos a un menor costo y de la 
necesidad que tiene la industria farmacéutica de generar productos con mayor valor agregado.

Hoy en día, las actividades para conocer el mercado de un producto tecnológico, su entorno competitivo, y decidir las estrategias de desarrollo se deben considerar no solo los análisis exclusivamente económicos. De acuerdo a Palop y Vicente (1999), la empresa debe tener la capacidad y la habilidad de percibir las señales indicadoras de cambios significativos en el entorno tecnológico, así como controlar día a día las actividades de importantes actores dentro y fuera de su sector. Así, la vigilancia tecnológica se presenta como una herramienta que permite analizar de manera organizada, selectiva y permanente la información que proviene del exterior de una unidad de desarrollo, convirtiéndola en conocimiento para tomar decisiones con menor riesgo y poder anticiparse a los cambios.(Palop y Vicente, 1999).

El presente trabajo se abordó como un estudio de caso para la aplicación de algunas herramientas de vigilancia tecnológica para determinar la posibilidad de producir medicamentos de base biotecnológica en Chile.

\section{Metodología}

La metodología busca primero detectar cuales son los biogenéricos potenciales de acuerdo a los antecedentes existentes en bases de datos nacionales e internacionales. Cabe mencionar que la concesión de una patente tiene carácter nacional, y en cada país, su legislación define los períodos de protección.

Para identificar los potenciales biogenéricos, se hizo una búsqueda de los biofármacos que expiraron sus patentes en el período 200I-2010, revisando las bases de datos de las oficinas de patentes Europea (http://ep.espacenet.com), de Estados Unidos de Norteamérica (http://www.uspto.gov) y de Chile (http://www.inapi.cl) y utilizando los códigos de clasificadores internacionales de patentes (CIP, versión 7) que describen productos biotecnológicos (OECD, 2005). Cabe mencionar que la Clasificación Internacional de Patentes, denominada habitualmente CIP, se basa en un tratado multilateral internacional administrado por la OMPI y denominado Arreglo de Estrasburgo relativo a la Clasificación Internacional de Patentes. En la práctica, la clasificación CIP la utilizan las oficinas de propiedad industrial de más de 100 estados, cuatro oficinas regionales y la Oficina Internacional de la OMPI. La clasificación es indispensable para la recuperación de los documentos de patente durante la búsqueda en el "estado de la técnica".

En paralelo, se deben reconocer los biofármacos que en Chile son de interés público, es decir, de primera necesidad. Para identificar los biofármacos de interés público en Chile se revisó el último listado oficial de medicamentos del Servicio Nacional de Salud, denominado Formulario Nacional de Medicamentos del año 2005 (Minsal, 2006).

También, se revisaron los biofármacos registrados en el Instituto de Salud Pública (www.ispch.cl) para tener una claridad respecto a cuáles de ellos son de interés en el mercado interno de Chile.

Para determinar el gasto de los biofármacos identificados en el Formulario Nacional se solicitó la información a la Central Nacional de Abastecimiento, CENABAST (www.cenabast.cl) y a las bases de datos de importaciones que maneja Prochile (www.prochile.cl).

El estudio cienciométrico se realizó de acuerdo a Vargas et al. (2006), para determinar los grupos de investigación en Chile que podrían tener la plataforma tecnológica necesaria para producir biogenéricos. Cabe indicar que la deducción de la plataforma se basa en las metodologías indicadas en las publicaciones científicas y no en un estudio de encuestas sobre el equipamiento tecnológico en los laboratorios reconocidos. Sin embargo, las publicaciones son altamente representativas de la capacidad tecnológica y en recursos humanos que presenta cada laboratorio de investigación. Aun así, existe en este análisis un sesgo no explorado, el cual corresponde a las empresas existentes en Chile que pueden tener plataformas tecnológicas, pero que en su mayoría no publican artículos cientíicos de sus trabajos.

La búsqueda y descarga de publicaciones científicas se realizó desde el sitio Web del National Library of Medicine and the National Institute of Health (NCBI, http://www.ncbi.nlm.nih.gov/). La estrategia de búsqueda se construyó en base a una o varias palabras claves.

\section{Resultados y Discusión}

\section{I El Mercado de los Biofármacos a Nivel Mundial}

Desde el año 1982, en el mundo se comercializan biofármacos. En USA, las ventas de las 5 mayores clases de 
proteínas usadas para preparar biofármacos exceden los US $\$ 22$ mil millones en el 2002 , con un total que se proyecta, alcanzará a los US $\$ 42.7$ mil millones en el año 2007, de acuerdo a un estudio realizado por Griffiths et al. (Griffiths, 2004).

Las ventas globales de los biofármacos alcanzaron los US $\$$ 56.2 mil millones en el 2004 y solo en USA, las ventas alcanzaron los US $\$ 30.8$ mil millones en el 2004 (PBR, 2006). También se prevé que para el año 2010, los biofármacos ocuparán el $35 \%$ del mercado de los productos farmacéuticos. Este aumento de la demanda, posiblemente no podrá ser cubierto por las actuales capacidades de producción, aumentando los precios de estos (Ruiz et al, 2005).

Los fármacos biotecnológicos, que apenas existían hace 15 años atrás, tuvieron un crecimiento medio en el 2003, del 15 por ciento, que corresponde al doble del crecimiento de los medicamentos convencionales. Tomando como ejemplo de biofármacos la Eritropoyetina (EPO), que venció su patente en el 2004, hasta el 2005 solo se encontraba en el mercado el producto de marca EPOGEN® (epoetin alfa), el cual aportó USD \$2,6 mil millones de ingresos a AMGEN (dueña de la patente) en el 2004. Sin embargo, hasta el 2005 existían más de 20 formas de biogenéricos de EPO que se encontraban en estado de desarrollo, la mayoría de los casos en fase preclínica (Charles, 2005).

Para el caso de la Hormona de Crecimiento Humano Recombinante, desde 1985 este biofármaco tuvo protección de patente perteneciente a Genentech, y solo recién en abril del 2006 se entregó una aprobación de EMEA (oficina técnica de medicamentos de la Unión Europea) a Sandoz (la división de genéricos de la biofarmacéutica suiza Novartis) para comercializar OMNITROPE, un biogenérico de la Hormona de Crecimiento Humana Recombinante (Tovar, 2006).

Cabe mencionar que cuando se selecciona un principio activo, ya sea éste, genérico o biogenérico, las farmacéuticas tradicionales y las biofarmacéuticas, compartirán el mismo criterio de selección, es decir, el que tenga el menor costo de producción y también de desarrollo. En la industria de los biofármacos este problema se ha resuelto mediante alianzas de investigación y fusiones entre compañías biotecnológicas y empresas farmacéuticas.
Por otro lado, las empresas que en estos momentos mantienen las patentes de los biofármacos, están haciendo más difícil la entrada de los biogenéricos, imponiendo las ventajas tecnológicas y de mercado, que en estos momentos hacen de ellas líderes, para mejorar las innovaciones por medio de nuevas patentes, de manera de mantener su competitividad. En este sentido, algunos autores hacen mención a los supergenéricos, como competencia de los biogenéricos (PBR, 2006). Así por ejemplo, Amgen para mantener liderazgo en el mercado, sustituyó su primer biofármaco, denominado Neupogen (factor estimulante de colonias de granulocitos, G-CSF) por otro de mejor comportamiento denominado Neulasta, reconvirtiendo en un $60 \%$ el mercado al nuevo producto, el cuál tiene una patente que lo protegerá hasta el 20132015. Esto define que solo una pequeña porción del mercado quedaría en manos de los posibles biogenéricos que pudieran aparecer.

En el caso de Eli Lilly, productora de Humalog, marca comercial de la insulina recombinante, no demuestran preocupación por los biogenéricos. Esta empresa que presenta un tercio de los 30 productos en desarrollo, basados en biotecnología acaba de inaugurar en el año 2006, una filial en Puerto Rico, para producir biofármacos. En el caso de las insulinas, se requiere una inversión de capital muy grande para producir grandes cantidades. Aún cuando existen producciones locales de insulina humana biosintética en diferentes partes del mundo, son operaciones con capacidad limitada y sólo pueden producir 25 kilos por año, contrastando con la demanda global de insulina, que es de miles de kilos. Además, por la complejidad de producir insulina, a los fabricantes de medicamentos genéricos se les hará sumamente difícil la producción de la misma a las escalas que demanda el mercado.

Sin embargo, las empresas de países menos desarrollados ya están en la búsqueda de alternativas para entrar en el mercado de los biogenéricos.

Biocon, una empresa india líder mundial en biotecnología genérica, ha utilizado el sistema de patentes para ser una de las primeras en tener acceso al sector de la producción de insulina humana. Cuando comenzaron, la patente sobre el producto a base de insulina humana había expirado hace mucho, pero todavía gozaba de protección mediante sólidas patentes de procedimientos. Biocon examinó todas las patentes publicadas que venían al caso, en búsqueda de 
un error que les permitiera entrar en el terreno. Se dieron cuenta que en la mayor parte de los procedimientos patentados se había utilizado $E$. coli y levadura de panadería, lo que los estimuló a probar otro tipo de levaduras y empezando a fabricar insulina utilizando levadura del genéro Pichia. Se trataba de un procedimiento nuevo y sin precedentes y que no había sido objeto de ninguna de las patentes en vigor.

La empresa India Wockhardt Ltd. es, a nivel mundial, el cuarto fabricante de insulinas de origen recombinante bajo las marcas Wosulinß- $\mathrm{N}$ (Insulina humana NPH isófona) y Wosulinß- R (Insulina humana de acción rápida) cuyo registro sanitario ha sido aprobado por el Instituto de Salud Pública de Chile. Tiene presencia en más de 90 países. Fresenius Medical Care AG (FMC AG) en fecha reciente firmó un acuerdo de comercialización a nivel mundial con Wockhardt Ltd. La sociedad Pentafarma S.A. es una filial en Chile de FMC AG de Alemania. Sin embargo, en Chile han recibido cuestionamientos respecto a la calidad de los productos (insulinas Wosulin $\mathrm{N}$ y Wosulin R), y a la legalidad del proceso de licitación convocado por la Central de Abastecimiento del Sistema Nacional de Servicios de Salud (Cenabast), en el cual se adjudicó a Pentafarma la provisión de insulinas para el sistema público de salud. Desde octubre de 2005, Pentafarma S.A. ha entregado al Sistema Público de Salud la insulina recombinante. Aquí se plantea quizás uno de los primeros conflictos de interés entre empresas de biogenéricos y biofarmacéuticas de marca en Latinoamérica.

Cabe hacer un punto aparte en el caso de algunos países latinoamericanos, como Argentina y Cuba que hace un largo tiempo están produciendo biogenéricos. A Chile, por ejemplo, se importa eritropoyetina Humana producida por la empresa Argentina Biosidus, de acuerdo a lo indicado en el registro de medicamentos del ISP. En este caso, Argentina ha sumado experiencia muy importante para comenzar a comercializar sus biogenéricos en Europa y Estados Unidos, en donde los mercados son mucho más grandes.

Desde 1990 en Argentina, Laboratorios Beta S.A. produce insulinas humanas con desarrollo tecnológico y científico argentinos. La empresa afirma que se han realizado exportaciones a algunos países de América Latina tales como Paraguay, Uruguay y México. En el año 2002 se comenzó a exportar a Estados Unidos
Lo mismo se aplica al caso de Cuba, quien produce biogenéricos en su Centro de Ingeniería Genética, y que en Chile son diferentes laboratorios los que importan sus productos.

Aquí cabe hacer mención que la biotecnología cubana ha producido un gran número de biofármacos, dentro de los cuales, el mas exitoso ha sido la vacuna contra la meningitis B, desarrollada en 1980, siendo la primera de su tipo en el mundo. Cuba comenzó su desarrollo biotecnológico imitando biofármacos tal como el Interferón alfa (2b). Hoy en día tienen cerca de 500 solicitudes de patentes basadas en mas de 200 innovaciones diferentes, en todo el mundo (Thorsteinsdóttir, H., 2004), lo cuál indica además que la estrategia del desarrollo de biogenéricos no solo crea el mercado de los biogenéricos, sino que promueve el desarrollo de nuevos productos biotecnológicos con innovaciones que pueden ser patentadas.

\subsection{Las Disposiciones Reglamentarias}

De acuerdo a Saavedra y Quiñones (2006) existe una serie de problemas asociados con los estudios de bioequivalencia (se refiere a la velocidad y proporción en que el mismo principios activos de dos medicamentos iguales alcanzan la circulación sistémica) en biogenéricos. La inmunogenicidad es una de las mayores preocupaciones en biofármacos, dado que especies alteradas conformacionalmente o pequeñas impurezas del proceso pueden conducir a una respuesta inmune que puede producir un efecto inhibitorio en la acción del medicamento o una respuesta alérgica. Un caso relevante ha sido el de Eprex, que corresponde a eritropoyetina $\alpha$, la que ha sido asociado a una aplasia (falta de crecimiento o definición de su estructura completa) de glóbulos rojos junto con producción de anticuerpos neutralizantes de eritropoyetina, lo que parecería estar relacionado con los cambios de formulación. Lamentablemente, hoy en día no existe un modelo animal validado para evaluar la inmunogenicidad de proteínas, motivo por el cual, la seguridad inmunológica debe ser probada en estudios clínicos y vigilancia posmercado (Saavedra y Quiñones, 2006).

Como resultado de la profundización de los análisis de bioequivalencia, se revocó la autorización de Omnitrop, la versión biogenérica de la hormona de crecimiento humano de Sandoz por parte de la Comisión Europea, a pesar de la recomendación positiva de la EMEA, su propio órgano

ISSN: 07I 8-2724. (http://www.jotmi.org)

Journal of Technology Management \& Innovation (@ Universidad Alberto Hurtado, Facultad de Economía y Negocios 
regulador en materia de medicamentos. La Comisión Europea decidió que estas copias no podrían aferrarse al medicamento original para demostrar su eficacia, sino que tendrían que realizar sus propios ensayos clínicos, tal y como lo hace cualquier fármaco innovador.

Omnitrope igualmente desarrolló estudios clínicos, demostrando que es equivalente al medicamento de referencia. Omnitrope se comparó con Genotropin en 89 niños con déficit de hormona de crecimiento que nunca habían recibido tratamiento. El estudio duró 9 meses y se midió la altura al principio y al final del mismo, así como la velocidad de crecimiento durante el estudio. Para evaluar la seguridad de Omnitrope, otros 51 niños han recibido también el medicamento durante un período de hasta un año. Tanto Omnitrope como Genotropin consiguieron incrementos similares en la altura y en la velocidad de crecimiento (equivalente a $+10,7 \mathrm{~cm} /$ año con ambos medicamentos). Se ha demostrado que la eficacia de Omnitrope es equivalente a la de Genotropin (EMEA, 2006).

Por su parte, la FDA (Food and Drug Administration) dice que será difícil aceptar versiones genéricas de biofármacos, debido a que ellos están basados en organismos biológicos complejos, mas que en moléculas simples, como son los medicamentos tradicionales (Rader, R., 2007).

Sin embargo, aún hay discusión sobre el tema, y la última palabra sobre la normativa todavía no está dicha. Mientras tanto, en el resto del mundo no esperan cuáles serán las definiciones de USA. Por ejemplo, la empresa israelí TEVA, la mayor productora de genéricos del Mundo y hoy dueña de Laboratorios Chile S.A., está construyendo plantas en México y Lituania para fabricar biogenéricos.

En Chile, el ISP (El Instituto de Salud Pública) recientemente ha regulado un sistema especial de registro de biofármacos que incluye ensayos clínicos de eficacia y seguridad así como farmacovigilancia post marketing. (Salinas y Becerra, 2007)

\subsection{Estudio de las Patentes en Estado de Convertirse en Biogenéricos a Nivel Internacional}

Aquí se indican los resultados de la vigilancia tecnológica de los documentos de la base de datos de patentes Europea (EPO) y de la Oficina de patentes y marcas de los estados Unidos (USPTO), para el período comprendido entre los años 1979-1990 (serían las patentes que expirarían entre los años 1999-2010), para los clasificadores seleccionados y que se indican en la metodología. Se inició la búsqueda utilizando el clasificador $\mathrm{Cl} 2 \mathrm{NOI} / 00$, que define a todas las patentes que involucran tecnologías de $\mathrm{ADN}$ recombinante.

El resultado del análisis indica que dentro del período 1980-1990 se encontraron 279 patentes publicadas relacionadas con productos de ADN recombinante (CI2NI5/00), 139 relacionadas con productos obtenidos por procesos de biosíntesis $(\mathrm{Cl} 2 \mathrm{P})$ y $1 \mathrm{I7}$ patentes relacionadas a clasificaciones relacionadas con microorganismos $(\mathrm{CI} 2 \mathrm{R})$.

Del conjunto de patentes relacionadas a biogenéricos, la empresa que más afectada se vería por esta situación corresponde a Genentech. Una búsqueda adicional de patentes en el buscador Patent Lens (www.patentlens.net), indica que esta empresa compromete 142 patentes en total, de las cuáles, 66 patentes están relacionadas con biogenéricos. Se hizo el análisis considerando que las patentes vencerían su protección en el año 2007.

\subsection{El Mercado de los Biofármacos en Chile}

En la industria farmacéutica chilena destacan 3 actores principales:

I) Los laboratorios farmacéuticos que en total suman 70 laboratorios y droguerías, en los que se cuentan diversos con capitales de origen extranjero. Entre los laboratorios nacionales, existen grandes laboratorios, que si bien no desarrollan moléculas, cuentan con modernas plantas productivas, que le permiten ocupar una posición de liderazgo, tanto en el mercado interno como en términos de exportaciones (Laboratorio Chile, Bagó, entre otros).

2) Las grandes cadenas de farmacias, cuyas ventas anuales dentro del país, se estiman en torno a los USD 600-650 millones, incluyendo sus líneas de medicamentos, cosméticos, perfumería, y otros. Las tres principales cadenas de farmacias, Farmacias Ahumada (Fasa), SalcoBrand y Cruz Verde, concentran aproximadamente un $90 \%$ de las ventas al detalle de productos farmacéuticos.

3) El sector público e institucional, cuyas compras anuales se estiman del orden de los US $\$ 120$ millones, 
aproximadamente US\$70 millones de los cuales son canalizadas a través de CENABAST.

Los medicamentos adquiridos por el sector público están dirigidos a intermediación de hospitales, a centros de atención primaria (consultorios), al Programa Nacional de Alimentación Complementaria y a Ventas Catálogo. Cabe hacer mención respecto a que en el año 2002, el ISP tomó la decisión de cerrar su departamento de producción de vacunas. Chile había tenido hasta esa fecha una tradición histórica de grandes aportes, como lo fue en su momento el desarrollo de la vacuna antirrábica, las que fueron debidamente fiscalizadas en su calidad y eficiencia. Sin embargo, según lo indicaron las autoridades del ISP que tomaron esta decisión en esa época, el departamento de producción de vacunas tenía serios problemas de financiamiento e infraestructura. Hoy en día, el Servicio Nacional de Salud paga mas de 3 mil 300 millones de pesos en comprar vacunas a los laboratorios privados. A principios del año 2007 se conoció la noticia del incumplimiento de contrato del Laboratorio Sanofi-Pasteur respecto a la entrega de las partidas de vacunas anti influenza que se requerían para la campaña de vacunación del año 2007, lo que le significó a este laboratorio que CENABAST hiciera efectivo el cobro de la boleta de garantía al proveedor.

Uno de los argumentos que hace pensar que la producción de vacunas debe ser de origen nacional tiene relación con que los productos importados son producidos con cepas que no necesariamente son las adecuadas para Chile y con estudios clínicos y de eficiencia desarrollados en otras poblaciones. Por lo que aquí se podría hacer un estudio respecto a la factibilidad de producir vacunas de origen recombinante de interés público.

\subsection{Los Biofármacos Existentes en Chile}

De acuerdo a la búsqueda de medicamentos obtenida de la base de datos del ISP, se encontraron 174 registros de biofármacos. De este listado se definieron los principios activos junto con sus funciones, que aparecen indicados en la tabla No. I.

\begin{tabular}{|c|c|c|}
\hline $\begin{array}{c}\text { Principio activo inscrito } \\
\text { en el ISP }\end{array}$ & Función & $\begin{array}{l}\text { Presente en el } \\
\text { formulario } \\
\text { nacional }\end{array}$ \\
\hline $\begin{array}{l}\text { Activador del plasminogeno } \\
\text { tisular humano recombinante }\end{array}$ & $\begin{array}{c}\text { I. Uso en Infarto del Miocardio (disolución } \\
\text { de coágulos), 2) Tratamiento de } \\
\text { hemorragias vítreas }\end{array}$ & NO \\
\hline $\begin{array}{l}\text { Aldesleukina-2 recombinante } \\
\text { (interleucina 2) }\end{array}$ & $\begin{array}{c}\text { Tratamiento de carcinoma renal metastásico, } \\
\text { del melanoma maligno avanzado, de la } \\
\text { leucemia aguda mieloblástica en recaída, } \\
\text { trasplante de médula ósea (autólogo y } \\
\text { alogénico). }\end{array}$ & NO \\
\hline Alefacept recombinante & $\begin{array}{c}\text { Tratamiento de la psoriasis refractaria a otras } \\
\text { terapias }\end{array}$ & NO \\
\hline $\begin{array}{l}\text { Antigeno de superficie dna } \\
\text { recombinante del virus de la } \\
\text { hepatitis b }\end{array}$ & Vacuna contra la Hepatitis B & NO \\
\hline $\begin{array}{l}\text { Desoxirribonucleasa humana } \\
\text { recombinante }\end{array}$ & $\begin{array}{l}\text { Aplicado en el Tratamiento de Fibrosis } \\
\text { Quística }\end{array}$ & NO \\
\hline
\end{tabular}

ISSN: 07/8-2724. (http://www.jotmi.org) 


\begin{tabular}{|c|c|c|}
\hline $\begin{array}{l}\text { Eritropoyetina (epoetin beta } \\
\text { recombinante) }\end{array}$ & $\begin{array}{l}\text { Tratamiento de la anemia asociada con } \\
\text { insuficiencia renal crónica }\end{array}$ & SI \\
\hline Estreptoquinasa recombinante & Tratamiento Infarto al Miocardio & NO \\
\hline $\begin{array}{l}\text { Factor de coagulacion } \\
\text { recombinante viia }\end{array}$ & $\begin{array}{l}\text { Tratamientos de hemorragias en Trastornos } \\
\text { como la hemofilia o el déficit de factor VII }\end{array}$ & SI \\
\hline $\begin{array}{l}\text { Factor de crecimiento } \\
\text { epidermico humano } \\
\text { recombinante (fce hum-rec) }\end{array}$ & Tratamiento Acné y úlceras cutáneas & NO \\
\hline $\begin{array}{l}\text { Factor recombinante humano } \\
\text { estimulador de colonias de } \\
\text { granulocitos y macrofago }\end{array}$ & $\begin{array}{l}\text { Tratamiento o profilaxis de la leucopenia } \\
\text { asociada con tratamientos mielodepresivos. }\end{array}$ & NO \\
\hline $\begin{array}{l}\text { Folitropina alfa recombinante } \\
\text { humana }\end{array}$ & Tratamiento Fertilidad Femenina & NO \\
\hline Folitropina beta recombinante & Tratamiento Fertilidad Femenina & NO \\
\hline $\begin{array}{l}\text { Glucagon clorhidrato (adn } \\
\text { recombinante) }\end{array}$ & $\begin{array}{l}\text { Hormona que eleva el nivel de glucosa } \\
\text { (azúcar) en la sangre }\end{array}$ & SI \\
\hline Infliximab- ca2 recombinante & Tratamiento de la enfermedad de Crohn & NO \\
\hline $\begin{array}{l}\text { Insulina humana (dna } \\
\text { recombinante) }\end{array}$ & Tratamiento de Diabetes & $\mathbf{S I}$ \\
\hline $\begin{array}{l}\text { Interferon alfa }-2 b \\
\text { (recombinante) }\end{array}$ & $\begin{array}{c}\text { Inmunomodulador, antiviral (heptitis B y C } \\
\text { crónica). }\end{array}$ & $\mathbf{S I}$ \\
\hline $\begin{array}{l}\text { Interferon alfa } 2 \mathrm{a} \text { humano } \\
\text { recombinante }\end{array}$ & Hepatitis B y Crónicas & NO \\
\hline $\begin{array}{l}\text { Interferon beta - la } \\
\text { recombinante }\end{array}$ & Tratamiento de Escrerosis Múltiple & NO \\
\hline $\begin{array}{l}\text { Interferon beta Ib humano } \\
\text { recombinante }\end{array}$ & Tratamiento de Escrerosis Múltiple & NO \\
\hline $\begin{array}{l}\text { Interferon gamma I - b } \\
\text { humano recombinante }\end{array}$ & $\begin{array}{c}\text { Tratamiento enfermedad granulomatosa } \\
\text { crónica }\end{array}$ & NO \\
\hline Interleukina - 2 recombinante & Tratamiento algunos tipos de Cancer & NO \\
\hline $\begin{array}{l}\text { Somatropina humana } \\
\text { recombinante (hormona del }\end{array}$ & Tratamiento en niños de estatura baja & NO \\
\hline
\end{tabular}




\begin{tabular}{|c|c|c|}
\hline crecimiento) & & \\
\hline Teriparatida recombinante & $\begin{array}{c}\text { Tratamiento de la osteoporosis en mujeres } \\
\text { posmenopáusicas con elevado riesgo de } \\
\text { fractura. }\end{array}$ & \\
$\begin{array}{c}\text { ( rhpth) }(\mathrm{I}-34, \text { hormona } \\
\text { paratiroidea humana) })\end{array}$ & & \\
\hline
\end{tabular}

Tabla I. Listado de los biofármacos registrados en el ISP.

Casi la totalidad de los principios activos que aparecen corresponden a hormonas peptídicas de no más de 40 aminoácidos y que no tienen estructura proteica cuaternaria, lo que hace más fácil su síntesis.

En la Tabla 2 se presenta el listado de los laboratorios farmacéuticos que registraron los principios activos biotecnológicos. Se observa que el principal laboratorio de biofármacos en Chile sería Laboratorio Bagó, con 19 registros, seguido de Laboratorio Bestpharma con 14 registros y Novo Nordisk, con 13 registros. El 76,4 \% de los registros corresponden a laboratorios internacionales y el resto a laboratorios nacionales. Sin embargo, como antes se mencionó, ningún biofármaco es producido en Chile, todos son importados terminados.

\begin{tabular}{|c|c|}
\hline Laboratorios & No. de registros \\
\hline Laboratorio Bago de Chile S.A. & 19 \\
\hline Laboratorio Bestpharma S.A. & 14 \\
\hline Novo Nordisk A/S Chile & 13 \\
\hline Novartis Chile S.A. & II \\
\hline Organon Chile Ltda. & I I \\
\hline Pentafarma S.A. & I I \\
\hline Schering Plough Cia. Ltda. & I I \\
\hline Eli Lilly Ltda. & 10 \\
\hline Pharma Investi De Chile S.A. & 10 \\
\hline Biolatina Chile S.A. & 9 \\
\hline Laboratorios Recalcine S.A. & 8 \\
\hline Tecnofarma S.A. & 7 \\
\hline Productos Roche Ltda. & 6 \\
\hline
\end{tabular}




\begin{tabular}{|l|c|}
\hline Aventis Pharma S.A. & $\mathbf{4}$ \\
\hline Pfizer Chile S.A. & $\mathbf{4}$ \\
\hline Glaxosmithkline Chile Farmaceutica Ltda. & $\mathbf{3}$ \\
\hline Laboratorios Raffo S.A. & $\mathbf{3}$ \\
\hline Pmg S.A. & $\mathbf{3}$ \\
\hline Abbott Laboratories De Chile Ltda. & $\mathbf{2}$ \\
\hline Boehringer Ingelheim Ltda. & $\mathbf{2}$ \\
\hline Laboratorio Biosano S.A. & $\mathbf{2}$ \\
\hline Laboratorio Volta S.A. & $\mathbf{2}$ \\
\hline Sanofi Pasteur S.A. & $\mathbf{2}$ \\
\hline Kirby Pharmaceuticals Ltda. & $\mathbf{I}$ \\
\hline Laboratorio Pasteur S.A. & $\mathbf{I}$ \\
\hline Laboratorios Andromaco S.A. & $\mathbf{I}$ \\
\hline Laboratorios D \& M Pharma Ltda. & I \\
\hline Merck S.A. & \\
\hline Schering De Chile S.A. & \\
\hline Teresa Ramelli Bertolotto & \\
\hline & \\
\hline
\end{tabular}

Tabla 2. Laboratorios que registran biofármacos en Chile

En el listado del año 2006 del formulario, aparecen descritos 12 biofármacos (Tabla 3), de acuerdo a lo analizado en este trabajo. Estos biofármacos, de acuerdo a la hipótesis, serían los biofármacos de interés público para el sistema nacional de salud.

\begin{tabular}{|c|c|}
\hline \multicolumn{2}{|c|}{ Nombre } \\
\hline Asparaginasa & Med.antineoplásico, citotóxicos \\
\hline Pegaspargasa & Med.antineoplásico, citotóxicos \\
\hline Interferon alfa (2b & Coadyuvante terapia oncológica \\
\hline
\end{tabular}

ISSN: 07I 8-2724. (http://www.jotmi.org) 


\begin{tabular}{|c|c|}
\hline recombinante) & \\
\hline Eritropoyetina & Antianémico \\
\hline Albumina humana & $\begin{array}{c}\text { Productos sanguíneos y sucedaneos del } \\
\text { plasma }\end{array}$ \\
\hline Factor VIII & $\begin{array}{c}\text { Productos sanguíneos y sucedaneos del } \\
\text { plasma }\end{array}$ \\
\hline Factor IX & $\begin{array}{c}\text { Productos sanguíneos y sucedaneos del } \\
\text { plasma }\end{array}$ \\
\hline Glucagón & Productos usados en diagnóstico \\
\hline Insulina humana Cristalína & Tratamiento de diabetes mellitus \\
\hline $\begin{array}{l}\text { Insulina Humana Isofana } \\
\text { (NPH) }\end{array}$ & Tratamiento de diabetes mellitus \\
\hline Insulina Ultrarápida & Tratamiento de diabetes mellitus \\
\hline Gonadotrofina Coriónica & Transtorno endocrino \\
\hline
\end{tabular}

Tabla 3. Biofármacos obtenidos del Formulario nacional

De este listado se pueden distinguir la eritropoyetina, la insulina y el interferón alfa, como los biofármacos libres de patentes hasta hoy. Estos productos por lo tanto, serían los biogenéricos de interés público en Chile y que podrían ser producidos. Sin embargo, también hay biofármacos que pronto expirarán su patente, como son los factores de coagulación VIII y IX.

\subsection{Las Patentes Asociadas a Biofármacos}

La eritropeyetina humana recombinante (epoetin) es una de las moléculas que ha perdido su protección de propiedad industrial. Esta molécula, para producirla hay que considerar algunas observaciones. Primero, la eritropoyetina es una macromolécula compleja, donde existe una parte proteica que es decisiva en la interacción con los receptores, y que contiene además modificaciones químicas de azúcar, que son susceptibles de variar dependiendo de diferentes factores (sistema de expresión, condiciones de cultivo, procedimientos de purificación pooling and downstream, etc.), y que afectan de manera importante la actividad biológica de la molécula in vivo (Trkulja, 2006).

Segundo, la eritropoyetina ha mostrado ser un principio activo prácticamente salvador de vidas, para pacientes con fallas crónicas renales, con alta eficiencia y muy alta seguridad ( Trkulja, 2006).

Tercero, el mercado mundial de la eritropoyetina se espera que sobrepase los 20 mil millones de dólares de USA, en unos pocos años. Claramente, una pequeña porción de este mercado podría ser interesante para cualquier compañía farmacéutica.

\subsection{Capacidades Tecnológicas en Chile}

Otro aspecto de interés corresponde a la capacidad tecnológica requerida para desarrollar un biogenérico.

En la Tabla 4 extraída de Bio....¿Qué? Biotecnología, el futuro llegó hace rato (Díaz, A., 2005) se presentan los pasos, operaciones y personal profesional involucrado en la producción de un biofármaco. Para el caso de un

ISSN: 07/8-2724. (http://www.jotmi.org)

Journal of Technology Management \& Innovation (c) Universidad Alberto Hurtado, Facultad de Economía y Negocios 
biogenérico, de acuerdo a la legislación existente, se requerirían las etapas 3-6 y 8 , si existiera un material biológico con la secuencia codificante de la proteína. Esto puesto que no se requeriría clonar dicho gen. Respecto a la etapa 7, solamente se requeriría del registro de un medicamento en la institución correspondiente, en Chile sería en ISP.

\begin{tabular}{|c|c|c|}
\hline Etapa & Personal & Operaciones \\
\hline I.- Investigación & Biólogo & Clonado/Expresión \\
\hline 2.- Fermentación/cultivos & Biotecnólogo & Selección de equipos y procesos \\
\hline 3.- Fermentación/Purificación & Ingenieros & Cambio de escala/Producción \\
\hline 4.- Bioanalítica & Químicos y Bioquímicos & Caracterizar Proteínas \\
\hline 5.- Galénica & Farmacéuticos & Formulación del medicamento \\
\hline 6.- Estudios Clínicos & Médicos & Estudios en Pacientes \\
\hline 7.- Registros-patentes & Abogados y Farmacéuticos & $\begin{array}{l}\text { Documentación para organismos de } \\
\text { control }\end{array}$ \\
\hline 8.- Lanzamiento al mercado & Personal Diverso & Ventas \\
\hline
\end{tabular}

Tabla 4. Etapas en la producción de un Biofármaco

Tomando cada una de las etapas como un clasificador del búsqueda, en la base de datos del NCBI, se generó la total de publicaciones encontradas con cada ecuación de siguiente tabla (Tabla 5) de resultados:

\begin{tabular}{|c|c|c|c|c|c|c|c|c|c|c|}
\hline ECUACIONES DEBÚSQUEDA & $\begin{array}{l}\text { TOTAL DE } \\
\text { ARTÍCULOS }\end{array}$ & 1 & 2 & 3 & 4 & 5 & 6 & 7 & 8 & $\begin{array}{c}\text { NO ES } \\
\text { BIOMEDICINA }\end{array}$ \\
\hline $\begin{array}{c}\text { Recombinant ANDprote in AND } \\
\text { chile [Affiliation] }\end{array}$ & 170 & 62 & 3 & 0 & 6 & 1 & 17 & 0 & 0 & 73 \\
\hline $\begin{array}{c}\text { recombinant } A N D \text { peptide } A N D \\
\text { chile [Affiliation] }\end{array}$ & 77 & 36 & 1 & 1 & 0 & 0 & 6 & 0 & 0 & 33 \\
\hline $\begin{array}{l}\text { Recombinant AND production } \\
\text { AND chile[Affiliation] }\end{array}$ & 20 & 14 & 2 & 0 & 0 & 0 & 0 & 0 & 0 & 5 \\
\hline $\begin{array}{c}\text { Bioreactor AND recombinant } \\
\text { AND chile[Affiliation] }\end{array}$ & 0 & 0 & 0 & 0 & 0 & 0 & 0 & 0 & 0 & 0 \\
\hline $\begin{array}{c}\text { Bioprocess AND recombinant } \\
\text { AND chile[Affiliation] }\end{array}$ & 0 & 0 & 0 & 0 & 0 & 0 & 0 & 0 & 0 & 0 \\
\hline $\begin{array}{c}\text { Foering AND protein AND } \\
\text { peptide AND chile[Affiliation] }\end{array}$ & 0 & 0 & 0 & 0 & 0 & 0 & 0 & 0 & 0 & 0 \\
\hline $\begin{array}{c}\text { Biotechnology AND } \\
\text { chile[Affiliation] }\end{array}$ & 44 & 3 & 2 & 5 & 0 & 0 & 0 & 0 & 0 & 40 \\
\hline
\end{tabular}

Tabla 5. Resultados de la Búsqueda Bibliográfica 
En ella se observa que la ecuación Recombinant and protein and Chile, generó el mayor número de artículos, sumando un total de 170. De éste total, 73 artículos fueron catalogados como artículos no relacionados con biomedicina. En este grupo se encuentran áreas de investigación en plantas, microorganismos ambientales, entre otros. Del total de artículos que sí están relacionados con biomedicina (97 publicaciones), 62 artículos correspondieron a resultados relacionados con la $I^{a}$ etapa de producción de un biofármaco, es decir, etapas relacionadas con identificación de genes, aislamiento, clonación y expresión de éstos en sistemas biológicos aplicados en escala de laboratorio.

Solamente 3 artículos se clasificaron en la $2^{\mathrm{a}}$ etapa de producción de un biofármaco, que corresponde a fermentación y cultivos celulares. Cabe mencionar en esta etapa a la investigadora de la Universidad Católica de Valparaíso, Claudia Altamirano, de la Escuela de Ingeniería Bioquímica, Facultad de Ingeniería, quién presentó artículos relacionados con el escalamiento de cultivo de células animales (células $\mathrm{CHO}$ ), plataforma tecnológica clave para la producción de proteínas recombinantes de origen recombinante. El grupo de investigadores liderado por C. Altamirano, desea desarrollar procesos de elaboración de la proteína recombinante Activador tisular del plasminógeno (t-PA) mediante la tecnología del cultivo de células mamíferas (células $\mathrm{CHO}$ ).

También en esta área se destaca J. Asenjo. Sin embargo, sus desarrollos están más enfocados a la biotecnología industrial con la producción de enzimas de uso industrial.

En la etapa 4 se encontraron 6 artículos relacionados, de los cuales I corresponde a N. Carvajal de la U. de Concepción. Este documento estudia el efecto del quelante EDTA (un compuesto utilizado en composiciones farmacéuticas) en la actividad de una Arginasa recombinante aislada de hígado humano $y$ que es producida en una $E$. coli.

Otra etapa importante, de acuerdo a los resultados indicados en la tabla 5 , es la que tiene relación con la etapa
6 o la relacionada con los estudios clínicos de biofármacos. Esta categoría obtiene el segundo lugar en el número de publicaciones con 17 artículos. Se destacan en este punto grupos de investigación tanto de universidades (principalmente grupos de la Universidad de Chile, Universidad de Concepción y universidad Católica. Pero también aparecen estudios realizados por profesionales de hospitales y clínicas. Esto es importante, porque se puede observar una oportunidad de desarrollar en base a esta experiencia, una plataforma de conocimiento y de infraestructura que pudiera sostener un servicio relacionado con estudios clínicos de biofármacos. (En Chile existe una empresa de capitales extranjeros dedicada a los estudios clínicos; ORGANON-Chile).

De acuerdo a los resultados, es evidente que en Chile no existen todas las capacidades tecnológicas necesarias para llevar un producto biotecnológico al mercado. Adicionalmente se puede indicar que actualmente muchos trabajos de investigación hablan de la baja productividad científica y de la escasa o casi nula productividad de creación tecnológica que existe en Chile (Brunner, 200I). Es sabido además, que incluso para importar tecnología, como sería el caso de los biogenéricos, se requiere de un recurso humano preparado para ello. Nuevamente es válido señalar la pobre inversión en I+D que realiza la industria farmacéutica nacional, la cual principalmente importa todos sus principios activos para preparar las composiciones farmacéuticas que requieren.

Haciendo una revisión de los proyectos financiados por CORFO y FONDEF relacionados con biomedicina que aparecen en el documento de CORFO, "Mapa de oportunidades en biomedicina" (http://www.corfo.cl/ index.asp?seccion=|\&id=3004), se observa que de 26 proyectos aprobados por CORFO y terminados en el período 1991-2006, ninguno corresponde al desarrollo de biofármacos de origen recombinantes, y solo 3 proyectos corresponden a bioconversiones de productos, para su uso en farmacología. Para el caso de los proyectos FONDEF, se encontraron 9 proyectos, 4 de los cuales están relacionados con biofármacos de origen recombinante. 


\begin{tabular}{|l|l|}
\hline Proyecto & Grupo realizador \\
\hline $\begin{array}{l}\text { Desarrollo de cepas bacterianas atenuadas como } \\
\text { vacunas orales vivas y producción de antígenos } \\
\text { recombinantes para inmunización contra } \\
\text { patógenos humanos y animales. }\end{array}$ & $\begin{array}{l}\text { Universidad Católica de Chile } \\
\text { Héctor Alejandro Venegas Esparza }\end{array}$ \\
\hline $\begin{array}{l}\text { Investigación y desarrollo de vacunas biológicas } \\
\text { para inmunoterapia oncológica. }\end{array}$ & $\begin{array}{l}\text { UNIVERSIDAD DE CHILE } \\
\text { Flavio Andres Salazar Onfray }\end{array}$ \\
\hline $\begin{array}{l}\text { Desarrollo y estandarización de tecnologías para la } \\
\text { produccion de anticuerpos monoclonales } \\
\text { modificados con aplicaciones en biomedicina. }\end{array}$ & $\begin{array}{l}\text { UNIVERSIDAD DE CHILE } \\
\text { Juan Carlos Aguillon Gutierrez } \\
\text { Fono: 6786725 } \\
\text { jaguillo@machi.med.uchile.cl }\end{array}$ \\
\hline $\begin{array}{l}\text { Generación de una vacuna recombinante contra el } \\
\text { virus respiratorio sincicial. }\end{array}$ & $\begin{array}{l}\text { UNIVERSIDAD CATOLICA DE CHILE } \\
\text { Alexis M. Kalergis } \\
\text { kalergis@bio.puc.cl }\end{array}$ \\
\hline
\end{tabular}

Tabla 6. Proyectos relacionados con biofármacos en Chile.

En definitiva, una empresa que desee ingresar en este mercado, debe adaptar sus capacidades técnicas para poder desarrollarlos, considerando que la base biotecnológica es muy distinta a la química. Si bien los resultados sobre vigilancia tecnológica de la producción científica chilena indican que no existen las plataformas tecnológicas suficientes para poder abarcar todas las etapas de producción de biogenéricos, no es menos cierto que la capacidad en recurso humano sí existe, pero no siempre está utilizado de la mejor manera para enfocarse a una problemática específica. Pues bien, aquí se puede abrir un importante lazo o vínculo entre las empresas farmacéuticas y las universidades o centros tecnológicos, que manejan plataformas tecnológicas y recursos humanos que son necesarios para la producción de un biofármaco. En este sentido, el sistema nacional de innovación tiene herramientas que permitirían al vínculo UniversidadEmpresa generar la transferencia tecnológica y el conocimiento para poder producir un biofármaco.

Por lo tanto, considerando que Chile aún no tiene todas las capacidades tecnológicas apropiadas para producir un biogenérico, pero que tiene un recurso humano capaz de incorporar nuevas capacidades, sería viable entonces pensar en producir un biofármaco que aún no está liberado de patente.

En definitiva, hoy en día la biotecnología está avanzando en casi todos los ámbitos de la farmacología, y prontamente el gran porcentaje de productos farmacéuticos serán de origen biotecnológico. Por ende, cualquier oportunidad de nuevos negocios en esta área debe ser evaluada.

De la información recogida se desprende que los biogenéricos en general son muy sofisticados y requieren para su elaboración, un largo período de gestión, de controles técnicos y clínicos precisos y sofisticados, junto con montos significativos de inversión en investigación y desarrollo.

A partir de los biofármacos registrados en Chile y las exportaciones del año 2006, se desprende que el $100 \%$ de los biofármacos importados son productos terminados y que no existe exportación de biofármacos desde Chile.

Se concluye que Chile aún no tiene suficiente capacidad tecnológica para abarcar todas las etapas que se requieren para colocar un producto biotecnológico en el mercado. Esto puede deberse a factores tales como la deficiencia en la obtención de capitales de riesgo específicos para el sector y la cultura imperante dentro de la comunidad científica, que no considera la vinculación con la empresa una actividad necesaria.

\section{Agradecimientos}

El autor agradece el aporte de Jorge Campos G. en la revisión de éste manuscrito y del profesor Jaime Olavarria de la Universidad de Talca que participó como profesor tutor de la tesis. 


\section{Bibliografía}

ARDER, R. (2007) What is a generic biopharmaceutical? Biogeneric? Follow-on protein? Biosimilar? Follow-on biologic? Bioprocess Internacional. Marzo 2007, 28-38.

BRUNNER, J.J. (200I) Chile: Informe sobre capacidad tecnológica. Programa de las Naciones Unidas para el desarrollo. Temas de desarrollo humano sustentable, 6 .

CHARLES, S. (2005) SuperGenerics: a better alternative for biogenerics. Drugs Discovery today 10 (8), 533-535. (www.drugsdiscoverytoday.com).

DOMINGUEZ-GIL, A. (2004). Las próximas EFG's, los biogenéricos, Editorial. Rev. Esp. Econ. Salud 3(3).

EMEA (2006). Informe público europeo de evaluación (EPAR) Omnitrope. Resumen del EPAR para el público general. European Medicines Agency, 12 de abril de 2006.

GRIFFITHS, S. (2004) Betting on biogenerics. Nature 3, 197-198.

HARRIS, P. (2007) Oportunidades y Desafíos para la Industria Farmacéutica. Business Chile, Informe especial. http://www.businesschile.cl/imprimir.php? $w=0 l d \& l a n=e s \& i d$ $=189$ (Visitado el 01/06/2007).

LEDERBERG, J. (2002) Top 10 biotechnologies for improving health in developing countries. Toronto, Program in Applied Ethics and Biotechnology (PAEB) and the Canadian Program on Genomics and Global Health (CPGGH). p. 132.

MINSAL (2006). Formulario nacional de medicamentos: Dto. $N^{\circ} 194$ de 2005. Ministerio de Salud, Depto. de Asesoría Jurídica. p. 22.

OECD (2005). A Framework for biotechnology statistics. Organization for economic co-operation and development.

OMPI (2005). La información sobre patentes: Un tesoro escondido. Revista de la OMPI, Enero/Febrero, 2005.

PALOP, F., Vicente, J. M. (1999) Vigilancia tecnológica e inteligencia competitiva, su potencial para la empresa española. COTEC Estudios, 15, II
PBR (2006) Biogenerics: The battle is only just beggining. Pharmaceutical Business Reviews On line. www.pharmaceutical-business-reviews.com (Visitado el 18 de enero 2006).

QUEZADA, F., Roca, W., Szauer, M., Gómez, J., López. R., (2005) Biotecnología para el uso sostenible de la biodiversidad. Capacidades locales y mercados potenciales. Informe preparado para la Corporación Andina de Fomento. CAF.

RUIZ, O., García, E., Gago, L., González, V. (2005). Plantas Biofactorías: Informe de Vigilancia Tecnológica. Madrid: Fundación Española para el desarrollo de la investigación en Genómica y Proteómica./Parque Científico de Madrid. p. 130.

SAAVEDRA, I., Quiñones, L. (2006). Intercambiabilidad de medicamentos de origen biológico (biofármacos): Consideraciones acerca de la aprobación de formulaciones biosimilares (biogenéricos) en Chile. Rev. Med. Chile. I34, I583-I588.

SALINAS, E., Becerra, F. (2007). Productos biológicos y biosimilares. Revista Diagnóstico, 46 (4), Octubre-Dic. 2007.

SCHELLEKENS, H., Ryff, J.C., (2002) 'Biogenerics': the offpatent biotech products. TRENDS in Pharmacological Sciences 23(3), II9-121.

THORSTEINSDÓTTIR, H., Sáenz, T.,Quach, U., Daar, A., Singer, P. (2004). Cuba-innovation through synergy. Nature Biotechnology 22, DC19-DC24.

TOVAR, M. (2006) Los biosimilares y el Futuro. http://diabetesstop.wordpress.com/2006/06/07/los-

biosimilares-y-el-futuro/ (Visitado el 25/09/2009)

TRKULJA, V. (2006). European Union Regulatory Draft Guidance on Biogenerics Containing Recombinant Human Erythropoietin. Croat Med J 47, I76-180.

VARGAS, P., Ortiz, I, Maturana, V. (2006). Vigilancia Tecnológica aplicada a Nanociencia y nanotecnología en países de Latinoamérica. J. Technol. Manag. Innov. I(4), 83-94. 